\section{A bad combination}

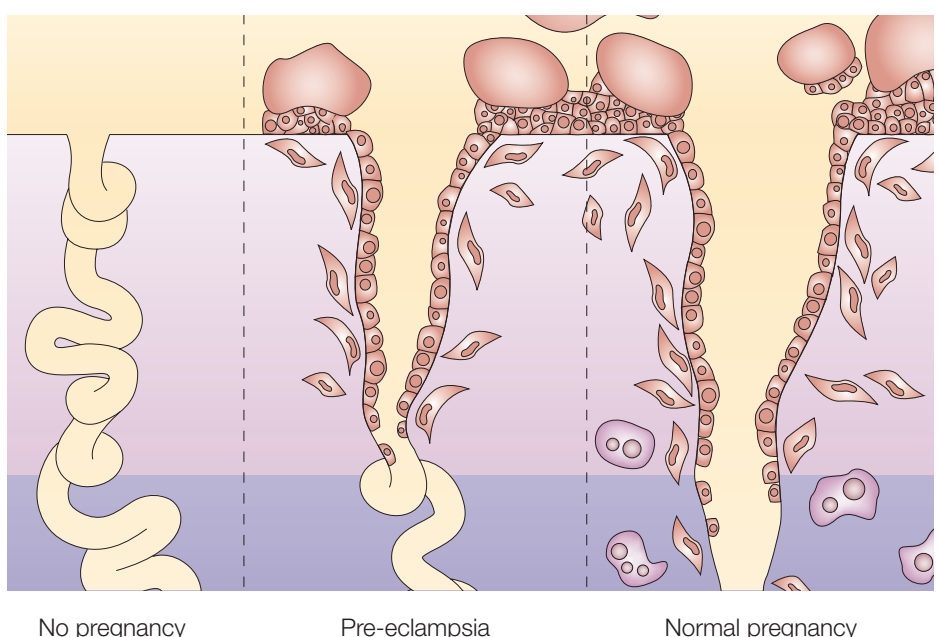

Pre-eclampsia is a complication of the second half of pregnancy, resulting in poor fetal growth and changes to the maternal systemic vasculature. About $5-10 \%$ of pregnancies are affected by pre-eclampsia, and it is a significant cause of pregnancyassociated mortality. The only cure is to deliver the fetus. Hiby and colleagues now report that a specific combination of maternal KIR (killercell immunoglobulin-like receptor) and fetal HLA-C genotypes is associated with an increased risk of preeclampsia.

In humans, placentation involves remodelling of the maternal blood vessels to provide the fetus with sufficient nutrients from the mother's blood supply. Maternal natural killer (NK) cells - which constitute $50-90 \%$ of the leukocytes in the decidua - have a role in remodelling the spiral arteries in the decidual layer. The activation of uterine NK cells, similar to all NK cells, is controlled by signals from both activating and inhibitory receptors. Previous studies have shown that maternal and paternal genes contribute to pre-eclampsia, and in this study, Hiby and colleagues chose to assess the maternal KIR genotype and the fetal HLA-C genotype because both gene families are polymorphic.

The authors analysed the frequency of maternal KIR and fetal HLA-C genotypes in 200 pregnant women with pre-eclampsia and 200 women with normal pregnancies. Pre-eclampsia was found to be more common in women homozygous for the KIR-A haplotype (denoted AA). This haplotype has seven KIR loci, only one of which is activating (KIR2DS4), and because the most common allele of KIR2DS4 has a deletion, women with an AA genotype generally have no activating KIRs. Each additional activating KIR decreased the risk of pre-eclampsia. When the combination of KIR and HLA-C genotypes in pre-eclampsia was assessed, the authors found that the combination of the KIR AA genotype with fetal HLA-C2 whether hetero- or homozygous was significant for the development of pre-eclampsia.

These results indicate that too much inhibition of uterine NK cells leads to inadequate remodelling of the maternal arteries and increases the likelihood of pre-eclampsia. So, this interaction between maternal KIR and trophoblast HLA-C2 supports a physiological function during placentation, rather than an immunological role in mounting a response to the allogeneic fetus.

Elaine Bell

(2) References and links ORIGINAL RESEARCH PAPER Hiby, S. E. et al. Combinations of maternal KIR and fetal HLA-C genes influence the risk of pre-eclampsia and reproductive success. J. Exp. Med. 200, 957-965 (2004).

\section{IN BRIEF}

\author{
APOPTOSIS
}

Kinase suppressor of Ras-1 protects intestinal epithelium from cytokine-mediated apoptosis during inflammation.

Yan, F. et al. J. Clin. Invest. 114, 1272-1280 (2004).

Inflammatory bowel diseases are characterized by altered cytokine production and increased intestinal epithelial-cell apoptosis. In this paper, Yan et al. show that kinase suppressor of RAS1 (KSR1) protects intestinal epithelial cells from apoptosis induced by tumour-necrosis factor (TNF). The authors observed that KSR1 and its target signalling pathways are activated in inflamed colonic mucosae, whereas KSR1 activity is turned off in normal mucosae. Moreover, KSR1-deficient mice had increased susceptibility to chronic colitis and TNF-induced epithelial-cell apoptosis. This was mediated by the kinase activity of KSR1, as expression of wildtype KSR1, but not kinase-inactive KSR1, restored TNF-induced activation of downstream mediators of anti-apoptotic pathways.

\section{INFLAMMATION}

Identification of bacterial muramyl dipeptide as activator of the NALP3/cryopyrin inflammasome.

Martinon, F., Agostini, L., Meylan, E. \& Tschopp, J. Curr. Biol. 14, 1929-1934 (2004).

Assembly of the inflammasome, a key component of which is NALP3, is triggered by ligation of Toll-like receptors (TLRs) with bacterial components, and this results in the activation of caspase-1 and the secretion of interleukin-1 $\beta$ (IL-1 $\beta$ ). Similar to TLRs, NALP3 contains leucine-rich repeats, which are frequently used to sense bacterial components. So, can bacteria directly activate the inflammasome? The authors found that bacterial peptidoglycan and its degradation product muramyl dipeptide (MDP), but not lipopolysaccharide, induced NALP3-mediated caspase-1 activation in a TLR-independent manner. Compared with macrophages from controls, macrophages from a patient with Muckle-Wells syndrome, an autoinflammatory disease associated with NALP3 mutations, showed increased IL- $1 \beta$ release in the presence of MDP, indicating that this pathway could be important in the disease.

\section{CYTOKINES}

Interleukin-12-induced interferon- $\gamma$ production by human peripheral blood $T$ cells is regulated by mammalian target of rapamycin (mTOR).

Kusaba, H. et al. J. Biol. Chem. 1 Nov 2004 (doi:10.1074/jbc.M405204200).

Interleukin-12 (IL-12) induces T-cell production of interferon- $\gamma$ (IFN- $\gamma$ ) by activating cyclosporin A-insensitive signalling pathways. In this paper, the production of IFN- $\gamma$ by pre-activated human peripheral-blood T cells in response to IL-12 was partially inhibited by another immunosuppressant, rapamycin. Rapamycin decreased IFN- $\gamma$ transcription, and this correlated with decreased recruitment of the transcription factors STAT3, STAT4 and phosphorylated cJUN. Serine phosphorylation of STAT3 was impaired, and its association with the serine/threonine kinase mammalian target of rapamycin (mTOR) was decreased. These observations led the authors to suggest that treatment of some diseases might require combination therapy to fully suppress T-cell function. 